\title{
SoundTag: \\ RFID Based Wearable Computer Play Tool for Children
}

\author{
Ryoko Ueoka $^{1}$, Hiroki Kobayashi ${ }^{2}$, and Michitake Hirose ${ }^{2}$ \\ ${ }^{1}$ Research Center for Advanced Science and Technology, the University of Tokyo, 4-11-16 \\ Komaba Meguro-Ku Tokyo, Japan \\ ${ }^{2}$ Cyber Interface Laboratory, the University of Tokyo, 7-3-1 Hongo Bunkyo-Ku Tokyo, \\ Japan \\ \{yogurt, hill_koba, hirose\}@cyber.t.u-tokyo.ac.jp
}

Societal demographics inform that the number of children is decreasing. Also there is a shortage of playgrounds around urban areas. These are factors that tend to influence the time children spend outdoors playing with friends. The decrease of outside playing time is unfortunate for children since outdoor activities contribute to children's well-being and development in ways that are not addressed by academic learning. Although there are many social reasons having decreased outside playing time, technological advancement such as television or computer games are considered to be one of the factors. However the authors suggest that the technological advancement is not the reason to cause this tendency. The trend of current computer games induces players doing physical activities indoors. In this study, we take the advantage of technological advancement into positive effect to motivate children playing outdoors. We propose SoundTag, which is a wearable computer prototype system to motivate them to play outside. It employs RFID tag and reader into wears to realize interactive play with sound feedback.

In this paper, we present the SoundTag concept, design theory and two types of wearable system and experiments. the first SoundTag system was applied to a play scenario, which is an enhanced version of a 'tag' game. We performed a pilot experiment partnered with children (from 5 years old to 10 years old) to observe how SoundTag affected playfulness. Playfulness is discussed from the observation of children while playing it and the comments from their parents after the experiment. Followed by the observations of the first experiment, the second version of the SoundTag system is deployed and is applied to the outdoor sound collection game. 